\title{
CONCLUSIONS
}

\author{
L. WOLTJER \\ Observatoire de Haute Provence, \\ F-04870 Saint Michel l'Observatoire, France
}

At the Albuquerque symposium in 1981, Jan Oort presented a general overview in which he listed a number of questions and tentative answers. The first five items in this summary are from his list.

\section{The source of energy}

Probably massive $\left(G M_{\odot}\right)$ black holes provide much of the energy, either through accretion or from their "rotational" energy. However, bursts of star formation appear also important in many cases and may contribute significantly. The possibility of extracting energy from binary black holes was also mentioned here; for the mechanism to be effective near relativistic binaries may have to be considered in which case gravitational radiation may pose a problem.

Much of the energy frequently emerges within a narrow cone

Originally focusing by pressure gradients was believed to be important, but now magnetic focusing is frequently invoked and may well be more effective. It is interesting that the Broad Absorption Line quasars (which may, in fact, be representative of the majority of quasars) show that a less focused subrelativistic flow is also possible. The $B A L$ quasars generally have no significant radio emission which could support the notion of magnetic focusing for radio quasars, but orientation dependent effects may also play a role. 
Jets are formed on many scales

At least close to the center these jets appear to be relativistic as evidenced by superluminal motions. This supports the belief that they originate in the environment of a black hole. In the less powerful (FRI) radio galaxies the jets appear to slow down to modest velocities $(0.01 \mathrm{c})$ at larger distances (kpc) from the center. The composition of the jets is still under debate with $e^{+} e^{-}$and $p e^{-}$plasmas being preferred under different circumstances. Shocks appear to be important in jets, which is confirmed by polarization observations which indicate that transverse magnetic fields occur frequently.

Numerical simulations have been rather successful in modelling the slowing down of jets by entrainment of surrounding matter. Filamentary structure and composite jets with a slower outer sheath appear in the simulations. Instabilities play an important role. Also jets with a continuous input of energy behave rather differently from those with an episodic input, because of the formation of multiple shocks. In this connection also the disappearance of some optical knots in the M87 jet during only a year seen by HST is of interest. In general there is a remarkable similarity in images of Herbig Haro jets $\left(100 \mathrm{Km} \mathrm{s}^{-1}\right.$ in interstellar matter), of jets around some galactic (few $M_{\odot}$ ) black holes and of extragalactic jets.

\section{Jets have a rather stable direction during millions of years}

Frequently this is ascribed to the stable rotation vector of the black hole; small changes in direction may be due to precessional motions. Some jets, especially at lower energies, are bent and this appears to be due to interaction with the gas in clusters of galaxies; this gas has become visible in X-ray observations.

\section{Superluminal motions}

At the Albuquerque meeting 7 cases of motions with apparent velocities in excess of that of light were reported. Now the sample includes 100 objects. The most plausible explanation is still relativistic motion at small angles to the line of sight, but the quantitative relation between bulk velocity of matter and pattern velocity is still unclear. One sided or very asymmetrical jets are then understood as due to Doppler effects. Support for such models come from observation of the Faraday rotation of some sources which indicates which side is directed toward us. The assumption is usually made that jets are intrinsically bisymmetrical, but it is still uncertain how much intrinsic asymmetry there is. 
In some cases when optical synchrotron radiation is seen in radio lobes the lifetime of the relativistic electrons is no more than $100-1000$ years. (Re)acceleration in situ is therefore needed and a Fermi-type shock acceleration is the most likely explanation. The resulting particle energy spectra are beginning to be understood.

\section{$X$-rays}

With Rosat some $10^{5}$ extragalactic X-ray sources have been detected, mainly AGN. Synchro-Compton models still appear to fit the gross characteristics. Of particular importance are $\mathrm{X}$-ray observations of the density and temperature of the gas in clusters, since from this the pressure in the radio lobes may be inferred. The non uniform temperature (ASCA) and the existence of important velocity fields show the clusters to be far more dynamic than previously thought. In some cases inverse Compton radiation from the lobes has been measured yielding values of the magnetic field, which sometimes are close to equipartition values. The fact that in the clusters the mass determined from the $\mathrm{X}$-ray data is about the same as that inferred from gravitational lensing gives confidence that the parameters of the gas can be believed.

\section{Gamma-rays}

Of the gamma-ray sources (with $|b| \geq 30^{\circ}$ ) observed with GRO/EGRET more than 2/3 have been identified with BL Lacs and OVV quasars with a very broad luminosity function. Since the gamma-ray background is rather weak more abundant objects like Seyferts and radioquiet quasars must be on average intrinsically faint in gamma-rays.

Synchro-Compton models with photons from the Broad Line Region have been presented; closer to the nucleus the $\gamma \gamma$ opacity would be too high for gammas to emerge. To quantify such models simultaneous optical, $\mathrm{X}$ and gamma data are needed with a resolution of less than a day. Such data may be correlated still in 1996 with GRO and later with "Spectrum $\mathrm{X}$, gamma" (1998) and Integral (2002).

\section{Unification with Doppler boosting}

If relativistic motions occur radio galaxies with jets pointing close to the line of sight could present the appearance of BL Lacs and it is therefore possible to "these two classes of objects. The situation is less clear with the OVV quasars which have emission lines with equivalent width only slightly 
smaller than ordinary quasars, while their $v / c$ values seem to be even larger than for BL Lacs.

\section{Unification with an absorbing torus}

In some Sy 2 and RG polarized broad emission lines (or heavily absorbed $\mathrm{X}$-rays) are seen which unambiguously indicate that Sy 1 or quasar-like nucleus is hidden behind absorbing matter. Statistical arguments in favour of unification on the basis of absorption effects are weaker. The observational results presented here on the diameters of quasars and radio galaxies appear still to be somewhat contradictory. In any case with models in which the opening angle of the torus is a function of power, age and redshift, there is so much freedom that definite conclusions about unification are not easy to come by.

A more direct way to study the effects of an absorbing torus is to observe in the IR where at some wavelength the torus should become transparent and the BLR visible. With ISO, the Infrared Space Observatory to be launched by ESA later this year, many AGN will be observed and the absorption effects quantitatively elucidated.

\section{Radiative ionization - Shock ionization}

In most cases it remains difficult to identify the ionizing mechanism. If radiative models do not work, optical depth effects and variability of the ionizing source may yield a fit to the emission line data. If simple shock models do not work multiple shocks, inhomogeneities and precursors can be invoked.

\section{Magnetic field structure}

The main distinction is between small scale tangled fields and larger scale flux carrying fields, the latter being needed to explain Faraday effects. Evidence was presented that Faraday effects originate in many cases outside the radio lobes in the cluster gas. The origin of the large scale fields there is ascribed to dynamo processes, but the details are far from clear. Accurate observations of polarization also in the visible with the new large telescopes should yield more definite information on the nature of the magnetic fields. 
High-z radio galaxies

Intriguing optical structures in these objects appear to be correlated with radio structures. The roles of AGN ionizing cones, shocks and star formation remain to be elucidated.

Some fraction of the numerous $\mu J y$ sources appears to have $z \geq 1$, but (post)starburst galaxies and AGN at lower redshifts also contribute to this population. With an estimated 20 sources per $\operatorname{arcmin}^{2}$ stronger than $1 \mu \mathrm{J} y$ there would be $3 \times 10^{9}$ such sources in the Universe, a significant fraction $(10 \%$ ?) of all galaxies.

\section{Gravitational Lenses}

These give the potentially important information on $\left(H_{0}, q_{0}\right)$, on intergalactic matter and on masses of galaxies and clusters, but accurate modeling is essential. With the lensing effects by clusters, galaxies and stars all superposed this is far from trivial. It is still uncertain how much the lensing affects the images of high-z galaxies.

\section{Evolution}

Most quasars are optically variable by more than $20 \%$ on time scales of 1 - 10 years, while global quasar lifetimes of $10^{7}-10^{8}$ years have been estimated. Nothing is known at intermediate time scales. Obviously, the simplest unified model would involve quasars switching on and off. Important variations in the BLR have been observed with PKS 0521-36 and BL Lac itself developing broad lines. Related variations have also been observed in quasars.

Systematic evolutionary effects (e.g. dust removal) have been suggested to relate hyperluminous infrared galaxies and quasars, while (with appropriate luminosity evolution included) the compact steep spectrum doubles could evolve into large radio galaxies. A certain gross evolutionary similarity is seen in the redshift distribution of radio galaxies, radio quasars and radio quiet quasars with a large increase in numbers towards $z=2$ and a steep decline for $z \geq 3-4$. For the radio objects this decline was shown not to be caused by absorption in the universe. A similar study in X-rays is needed to confirm the same conclusion for radio quiet quasars.

\section{Mergers}

There is a certain belief that AGN are generally related to galaxy mergers and in many cases the evidence looks very strong. At the same time there 
is much uncertainty if most $\mathrm{gE}$ galaxies are really the result of mergers, in particular because of arguments involving globular cluster frequencies.

\section{Why ellipticals?}

As at Albuquerque, we are not yet able to answer the most conspicuous question: what is so special about $\mathrm{gE}$ galaxies that all powerful radio galaxies in our neighborhood originate from these and never from spirals?

\section{The future}

VSOP and Radio Astron (1996/98) will improve the resolution of VLBI. Image quality may benefit, too. This is also important for the reliable measurement of the proper motions of features with changeable shapes.

ISO (1995) will tell us how frequent BLR's are in radio galaxies and Sy 2's. It also will contribute to the calorimetry of AGN by allowing a better evaluation of the energy radiated by dust.

HST (1997) will acquire a near infrared capability $(\leq 2.5 \mu m)$ which should allow intermediate and high-z radio galaxies to be observed with less trouble due to dust.

Large samples of radio and $\mathrm{X}$-ray sources $\left(10^{5}-10^{6}\right)$ are becoming available, the former from NRAO, Parkes and Westerbork, the latter from ROSAT. Optical identifications and redshifts are lagging behind. With $10 \%$ of the 4-m telescope time available in the word a sizeable part could be dealt with in the coming decade. Cooperative arrangements would be desirable.

Simulations of the complex systems involved in the extragalactic radio sources will remain very necessary and hopefully will lead to a more global understanding.

Radio galaxies were first identified in 1949 and quasar redshits in 1963. By the time of the Albuquerque meeting in 1981, a general knowledge of identifications, evolution and energetics existed. Jets and superluminal motions had been detected and the first gravitational lens identified. In the meantime, we have obtained many new data: instead of mJy sources we now discuss $\mu J y$ sources, instead of a few hundred X-ray sources we now have more than $10^{5}$. Instead of one uncertain gamma-ray AGN we now have several dozens. However, we still do not have a satisfactory answer to the simple question as to what makes an extragalactic radio source. 